INPLASY

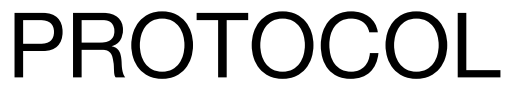

To cite: Mei et al. Meditation Programs for Adults with Type 2 Diabetes Mellitus: Protocol for a Systematic Review and Meta-analysis. Inplasy protocol 2021100008. doi:

10.37766/inplasy2021.10.0008

Received: 02 October 2021

Published: 02 October 2021

Corresponding author:

Manxue Mei

meimanxue13@163.com

Author Affiliation:

The Artemisinin Research Center, Guangzhou University of Chinese Medicine, Guangzhou, China; The Second Clinical College, Guangzhou University of Chinese Medicine,Guangzhou, China.

Support: Guangdong Province Key Field.

Review Stage at time of this submission: The review has not yet started.

Conflicts of interest:

None declared.

\section{Meditation Programs for Adults with Type 2 Diabetes Mellitus: Protocol for a Systematic Review and Meta-analysis}

\author{
Mei, M1; Jiang, M²; Li, Z3; Zhu, W4; Song, J5.
}

Review question / Objective: Would meditation programs affect fasting blood glucose levels and $\mathrm{HbA}(1 \mathrm{c})$ of patients with type 2 diabetes mellitus? Would meditation programs intervention be of benefit for remission of depression and anxiety level? Would meditation programs improve quality of life of individuals with type 2 diabetes? Do meditation programs affect body mass index (BMI), serum lipid levels and level of blood pressure? Which type of meditation programs is better for type 2 diabetes patients? Are there any differences of efficacy among different meditation programs? To provide valid evidence for the effect of meditation programs for type 2 diabetes by synthesizing and comparing outcomes from clinical trials.

Main outcome(s): The outcomes include fasting blood glucose levels and $\mathrm{HbA}(1 \mathrm{c})$.

INPLASY registration number: This protocol was registered with the International Platform of Registered Systematic Review and Meta-Analysis Protocols (INPLASY) on 02 October 2021 and was last updated on 02 October 2021 (registration number INPLASY2021100008).

\section{INTRODUCTION}

Review question / Objective: Would meditation programs affect fasting blood glucose levels and $\mathrm{HbA}(1 \mathrm{c})$ of patients with type 2 diabetes mellitus? Would meditation programs intervention be of benefit for remission of depression and anxiety level? Would meditation programs improve quality of life of individuals with type 2 diabetes? Do meditation programs affect body mass index (BMI), serum lipid levels and level of blood pressure? Which type of meditation programs is better for type 2 diabetes 
patients? Are there any differences of efficacy among different meditation programs? To provide valid evidence for the effect of meditation programs for type 2 diabetes by synthesizing and comparing outcomes from clinical trials.

Condition being studied: According to the International Diabetes Federation (IDF) Diabetes Atlas (9th edition) published by WHO, nearly 4.63 million adults aged between 20 to 79 suffer from diabetes mellitus (DM) globally, and this number is projected to reach 578 million by 2030 , and 700 million by 2045. T2DM could trigger numerous complications, including microvascular, macrovascular complications and diabetic neuropathies, and increases the risk of stroke, cognitive impairment and cancer. A survey from IDF indicates that the global economic burden of diabetes-related diseases is around US $\$ 673$ billion in 2015 . The development of T2DM is closely associated with low-level, chronic inflammation, insulin resistance and various metabolic disorders. Human lifestyle alterations have contributed to a rapid increase of T2DM worldwide. Demand of modern life makes people suffer from chronic stress for a long time. Chronic stress could cause excitement of sympathetic nerve, leading to activation of the hypothalamus-pituitary-adrenal gland (HPA). The activation of HPA could cause the release of glucocorticoids and cortisol, affecting body's blood sugar homeostasis, which could finally cause insulin resistance. Additionally, there is currently considerable evidence of an association between negative stress (like depression and anxiety) and T2DM. DM is reciprocally linked to depression through many ways. Researches have proved that depression may make the body in a low-grade inflammation state, and C-ractive protein (CRP), as an inflammatory-related factors, partially mediates the relationship between depression and diabetes. Negative emotional state could be changed by mindbody therapies. As a non-drug approach for T2DM, mind-body therapies have gained more attention than ever before, which could also help reduce social medical costs. Meditation is a kind of mind- body interventions, a form of metal training that helps individuals train their attention and awareness to achieve mental well-being. Meditation has many forms, like transcendental meditation and mindfulness meditation.Meditation may benefit to other stress-related medical diseases, such as COPD, hypertension, and chronic pain . In 2013, the American Heart Association (AHA) has recommended transcendental meditation as an alternative therapy for hypertension with level Ilb evidence. Meditation could reduce many indicators related to the metabolic syndrome, such as blood pressure, blood lipid levels, blood sugar level, glycosylated hemoglobin and BMI. Clinical trials have found that meditation programs could also affect HbA1c level and improve quality of live of T2DM patients. Though the mechanism of meditation for T2DM is not clear, some studies have found that meditation programs may improve symptoms of T2DM patients through many ways. The development and progression of T2DM is associated with the dysfunction of HPA axis, which could be regulated by meditation. A study found that emerging adults trained with transcendental meditation have lower morning cortisol level and cortisol awakening response. Inflammation plays an important role in T2DM. A meta-analysis in 2017 has summarized the influence of meditation for T2DM, and the results show that mindfulness meditation could reduce the activity of nuclear factor kappa-light-chainenhancer of activated B cells (NF-kB), lower the level of $\mathrm{C}$-reactive protein (CRP) and increase the level of CD4+. Some researches have confirmed that meditation programs have positive impacts on the length of telomeres and reduce the reactive oxygen species (ROS), and reduce the development of DM related to aging. Now, meditation has received more attention among people. There are also a number of clinical trials exploring the impact of various meditation programs on T2DM patients and gained considerable results . But discrepancies also exist in these studies and there is a lack of lateral 
contrasts among researches related to different meditation programs.

\section{METHODS}

Search strategy: Seven databases will be searched from 1990 to 2021 in Chinese and English with no publication restricts. The English databases include Embase, PubMed, Cochrane Library and Ovid database. Meanwhile, the Chinese databases will be searched, including China Science and Technology Journal Database, Chinese Biomedical Literature Database, China National Knowledge Infrastructure (CNKI) and Wan-fang Database, VIP information database. Three independent reviewers (MMX, LZJ, JM) will finish this job. $\mathrm{P}+\mathrm{I}+\mathrm{C}+\mathrm{O}+\mathrm{S}, \mathrm{P}+\mathrm{I}+\mathrm{C}+\mathrm{O}$ and $P+I+O$ will be used to detail the main elemetns of searched topics. The followings are details of topics. $P$ (Participants) : Type 2 diabetes mellitus; Type 2 diabetes; Type II diabetes; Type II diabetes mellitus; T2DM; insipidus, non insulin dependent diabetes mellitus; I (Interventions) : Meditation; Transcendental meditation; Mindfulness meditation; Mindfulness; Tai Chi; yoga; C (Controls) : Placebo treatment, treatment as usual or with no treatment; $O$ (Outcomes) : Major outcomes: Fasting blood glucose levels and HbA(1c); Secondary outcomes: remission of depression and anxiety level, quality of life, BMI, blood pressure, serum lipid levels; S (Study types): Random clinical trials(RCTs), controlled clinical trials(CCTs).

Participant or population: Adults diagnosed with T2DM are included in this study. In this review, the definition for T2DM should follow relevant guidelines, such as American Diabetes Association standard for clinical diagnosis and treatment of diabetes.

Intervention: Our protocol contains researches taking different forms of meditation as treatment, whether as a primary or complementary therapy. It also includes some other comprehensive therapies including meditation, such as yoga and Tai Chi.
Comparator: Placebo treatment, treatment as usual or no treatment.

Study designs to be included: Random clinical trials(RCTs) or controlled clinical trials(CCTs).

Eligibility criteria: ParticipantsAdults diagnosed with T2DM are included in this study. In this review, the definition for T2DM should follow relevant guidelines, such as American Diabetes Association standard for clinical diagnosis and treatment of diabetes.Interventions Our protocol contains researches taking different forms of meditation as treatment, whether as a primary or complementary therapy. It also includes some other comprehensive therapies including meditation, such as yoga and Tai Chi .ControlsPlacebo treatment, treatment as usual or no treatment.OutcomesThe main outcomes will be fasting blood glucose levels and $\mathrm{HbA}(1 \mathrm{c})$. The second outcomes are remission of depression and anxiety level, quality of life, BMI, highdensity lipoprotein cholesterol, low-density lipoprotein (LDL) and level of blood pressure.Study typesThis study includes Random clinical trials(RCTs), controlled clinical trials(CCTs), while case studies, conference papers, review, comments, retrospective cohort studies, cluster trials, cross-sectional studies are excluded.

Information sources: Our information comes from several databases. The English databases include Embase, PubMed, Cochrane Library and Ovid database. Meanwhile, the Chinese databases will be searched, including China Science and Technology Journal Database, Chinese Biomedical Literature Database, China National Knowledge Infrastructure (CNKI) and Wan-fang Database, VIP information database. And we will also contact with authors to gain more information unpunished.

Main outcome(s): The outcomes include fasting blood glucose levels and $\mathrm{HbA}(1 \mathrm{c})$.

Additional outcome(s): The second outcomes are remission of depression and 
anxiety level, quality of life, BMI, highdensity lipoprotein cholesterol, low-density lipoprotein (LDL) and level of blood pressure.

Quality assessment / Risk of bias analysis: Cochrane Risk of Bias tool will be used to assess random sequence generation, allocation concealment, blinding of participants and personnel, blinding of outcome assessment, incomplete outcome data, selective reporting and other bias of all included studies. Then make judgment for every studies with low risk, unclear risk and high risk. And Newcastle-Ottawa-Scale (NOS) will be used to evaluate studies which are not RCTs. The second outcomes are remission of depression and anxiety level, quality of life, BMI, high-density lipoprotein cholesterol, low-density lipoprotein (LDL) and level of blood pressure.

Strategy of data synthesis: All statistical analyses will be performed using Revman5.3.0 (Cochrane information management system) and Stata V.16.0 software. For continuous outcomes, we will calculate mean differences (MDs) with 95\% Cls and 12 statistic for heterogeneity across studies. A value of $\mathrm{P} 0.1,12<50 \%$ ), fixed-effect model will be used. Random effect model would be used to analyze when there was heterogeneity among the studies ( $P \leq 0.1,12>50 \%$ ).

Subgroup analysis: Subgroup metaanalyses and meta-regressions are conducted to explore reasons of heterogeneity, such as types of study design, populations and different meditation programs.

Sensitivity analysis: We will also perform a sensitivity analysis by excluding studies with high risk of bias.

\section{Country(ies) involved: China.}

Keywords: Meditation; type 2 diabetes mellitus; $\mathrm{HbA}(1 \mathrm{c})$; adults; quality of life; BMI; HDL; LDL; blood pressure.
Contributions of each author:

Author 1 - Manxue Mei - The author designed this protocol and drafted the manuscript.

Email: meimanxue13@163.com

Author 2 - Min Jiang.

Email: 18482102428@163.com

Author 3 - Zunjiang Li.

Email: 870188404@qq.com

Author 4 - Wei Zhu.

Email: zhuwei9201@163.com

Author 5 - Jianping Song.

Email: songjianping@sina.com 